\title{
Optimization of relay placement and power allocation for decode-and-forward cooperative relaying over correlated shadowed fading channels
}

\author{
Liang Han ${ }^{*}$, Jiasong Mu, Wei Wang and Baoju Zhang
}

\begin{abstract}
In this paper, we investigate the relay placement and power allocation for decode-and-forward (DF) cooperative relaying over correlated lognormal shadowed Rayleigh fading channels. Assuming that maximum-ratio combining is performed at the destination, we first derive an upper bound of symbol error rate (SER) with $M$-phase-shift keying (PSK) modulation. Then, three optimization problems are formulated to minimize the obtained SER upper bound, namely optimal relay placement with fixed power allocation, optimal power allocation with fixed relay location, and joint optimization of relay placement and power allocation. It is shown by the analytical results that the correlation coefficients and the standard deviations of shadowing have significant impacts on the optimal relay placement and power allocation. The simulation results validate our analysis and show that the SER upper bound is asymptotically tight in the high signal-to-noise ratio (SNR) regime.
\end{abstract}

Keywords: Correlated shadowing; Decode-and-forward; Outage probability; Power allocation; Relay placement

\section{Introduction}

Recently, the concept of cooperative relaying has been adopted in next-generation mobile communication standards, such as WiMAX and LTE-Advanced [1,2]. In these systems, fixed relays are deployed as intermediate nodes to forward data between mobile users and base stations (BS), thus improving the transmission reliability and extending the network coverage [3,4].

Although the performance analysis and optimization for various cooperative relaying schemes, such as amplifyand-forward (AF) and decode-and-forward (DF), have been extensively studied (see, e.g., [3-8] and references therein), most of the current works are mainly limited to the cases that only consider the small-scale fading, e.g., Rayleigh, Rician, or Nakagami fading, and few works have worked on the cooperative relaying systems considering the large-scale fading. The large-scale fading, also

*Correspondence: hanliang_tinu@163.com

College of Electronic and Communication Engineering, Tianjin Normal University, Tianjin 300387, China known as shadowing, is caused by obstacles, e.g., buildings and hills, between the transmitter and receiver, and often modeled by a lognormal distribution. In realistic environments, the source, relay, and destination are distributed in different locations, and thus, the effects of shadowing between any two of them are more likely to be different and correlated, which has also been demonstrated by empirical measurements [9,10]. In [11], the authors investigated the bit error rate of single-relay AF relaying over lognormal channels. The performance analysis of multi-hop AF cooperative relaying systems over lognormal channels was investigated in [12]. In [13], the outage performance of DF relaying over correlated lognormal channels was investigated. However, these papers were limited to the channels of pure lognormal distribution, and they have not considered the composite fading channels which include small- and large-scale fadings (i.e., correlated shadowed fading channels).

Our contribution in this paper can be briefly described as follows. First, we derive an upper bound of symbol error rate (SER) for DF cooperative relaying over correlated

\section{Springer}

(c) 2014 Han et al: licensee Springer. This is an Open Access article distributed under the terms of the Creative Commons Attribution License (http://creativecommons.org/licenses/by/4.0), which permits unrestricted use, distribution, and reproduction in any medium, provided the original work is properly credited. 
lognormal shadowed Rayleigh fading channels with $M$ phase-shift keying (PSK) modulation, which shows how the correlation coefficients and the standard deviations of shadowing impact the SER performance. Second, by minimizing the obtained SER upper bound, we formulate and compare three schemes to optimize the relay placement and power allocation. The simulation results show that joint optimization obtains the best SER performance and the SER upper bound is asymptotically tight in the high signal-to-noise ratio (SNR) regime.

The rest of the paper is organized as follows. In Section 2, the system model is introduced. We derive the upper bound of SER in Section 3 and formulated three optimization problems with an aim to minimize the upper bound of SER in Section 4. Afterward, in Section 5, we present some numerical and simulation results to validate our analysis. Finally, Section 6 concludes this paper.

\section{System model}

Consider a three-node relay channel, where a source communicates with a destination through a single relay as depicted in Figure 1. The DF cooperative relaying scheme adopted in this paper is carried out in two phases. In the first phase, the source transmits a signal to the relay and the destination, and the received signals at the relay and the destination are given as

$$
\begin{aligned}
& y_{\mathrm{sr}}=\sqrt{P_{s} l_{\mathrm{sr}}^{-\alpha} \xi_{\mathrm{sr}}} h_{\mathrm{sr}} x+n_{\mathrm{sr}}, \\
& y_{\mathrm{sd}}=\sqrt{P_{s} l_{\mathrm{sd}}^{-\alpha} \xi_{\mathrm{sd}}} h_{\mathrm{sd}} x+n_{\mathrm{sd}},
\end{aligned}
$$

respectively, where $P_{s}$ is the source transmit power, $\alpha$ is the path loss exponent [14], and $x$ denotes the transmitted symbol with unit power, i.e., $E\left\{|x|^{2}\right\}=1$ with $E(\cdot)$ representing the expectation operation. The relay decodes its received signal and checks if it is correct, which can be done by examining the SNR or checking the cyclic redundancy check (CRC) codes. If the relay decodes the received symbol successfully, it forwards the decoded symbol to the destination in the second phase; otherwise, it keeps silent. Then, the received signal at the destination from the relay is given as

$$
y_{\mathrm{rd}}=\sqrt{\tilde{P}_{r} l_{\mathrm{rd}}^{-\alpha} \xi_{\mathrm{rd}}} h_{\mathrm{rd}} x+n_{\mathrm{rd}},
$$

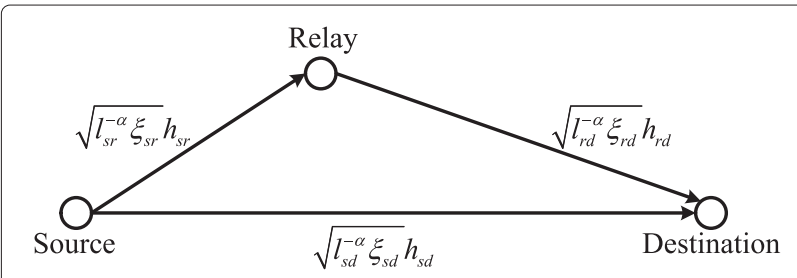

Figure 1 Channel model for the three-node relay channel. with $\tilde{P}_{r}$ being the transmit power at the relay. If the symbol is correctly decoded, $\tilde{P}_{r}=P_{r}$; otherwise, $\tilde{P}_{r}=0$. Here, $l_{\mathrm{sr}}, l_{\mathrm{sd}}$, and $l_{\mathrm{rd}}$ denote the distances of the source-relay, source-destination, and relay-destination links, respectively; $h_{\mathrm{sr}}, h_{\mathrm{sd}}$, and $h_{\mathrm{rd}}$ are the small-scale fading coefficients of the source-relay, source-destination, and relaydestination links, respectively, which are assumed to be independent and identically distributed (i.i.d.) zero-mean complex Gaussian random variables with unit variance; $\xi_{\text {sr }}, \xi_{\text {sd }}$, and $\xi_{\text {rd }}$ capture the shadow fading effects of the source-relay, source-destination, and relay-destination links, respectively, which are assumed to be lognormal random variables [14], i.e., $10 \log _{10}\left(\xi_{\text {sr }}\right), 10 \log _{10}\left(\xi_{\text {sd }}\right)$, and $10 \log _{10}\left(\xi_{\text {rd }}\right)$ are Gaussian with zero mean and standard deviation $\sigma_{\mathrm{sr}}, \sigma_{\mathrm{sd}}$, and $\sigma_{\mathrm{rd}}$, respectively; $n_{\mathrm{sr}}, n_{\mathrm{sd}}$, and $n_{\text {rd }}$ denote i.i.d. complex additive white Gaussian noise (AWGN) with zero mean and variance $N_{0}$.

The assumption of i.i.d. small-scale fading is reasonable since all nodes are separately deployed far away from each other. However, measurements in some practical mobile communication environments have shown that the lognormal shadowings are usually correlated [9]. In general, there are two types of shadowing correlation. The first type of correlation, often referred to as auto-correlation, represents the correlation between two links with a common start node and different end nodes. Correspondingly, the second type of correlation, often referred to as cross-correlation, represents the correlation between two links with a common end node and different start nodes [10]. As such, the shadowing between the source-destination and source-relay links is autocorrelated, and that between the source-destination and relay-destination links is cross-correlated. Denote the correlation coefficient between $10 \log _{10}\left(\xi_{\mathrm{sd}}\right)$ and $10 \log _{10}\left(\xi_{\text {sr }}\right)$ as $\rho_{1}$ and that between $10 \log _{10}\left(\xi_{\text {sd }}\right)$ and $10 \log _{10}\left(\xi_{\text {rd }}\right)$ as $\rho_{2}$. Besides, the shadowing between the source-relay and relay-destination links is assumed to be uncorrelated, which has also been demonstrated in [9].

We assume that the source and the relay have the statistical information of shadowing, and the destination has perfect channel state information. Then, the optimal processing at the destination is maximal-ratio combining (MRC) for the received signals from the first and second phases, and the output SNR is given as [3]

$$
\gamma=\frac{P_{s} l_{\mathrm{sd}}^{-\alpha} \xi_{\mathrm{sd}}\left|h_{\mathrm{sd}}\right|^{2}+\tilde{P}_{\mathrm{r}} l_{\mathrm{rd}}^{-\alpha} \xi_{\mathrm{rd}}\left|h_{\mathrm{rd}}\right|^{2}}{N_{0}}
$$

\section{SER upper bound}

In this section, we analyze the SER performance for the considered relay channel. For simplicity, we assume that all links use $M$-PSK modulation fordata transmission 
where $M$ is the size of the constellation, and the SER for the instantaneous SNR $\gamma$ is given by [15]

$$
P_{e}(\gamma)=\frac{1}{\pi} \int_{0}^{(M-1) \pi / M} \exp \left(-\gamma \frac{\sin ^{2}(\pi / M)}{\sin ^{2} \theta}\right) d \theta
$$

Using the law of total probability, the SER for the DF cooperative diversity is given as

$$
\begin{aligned}
P_{e}\left(\gamma, \gamma_{\mathrm{sr}}\right)= & \left.P_{e}(\gamma)\right|_{\tilde{P}_{r}=0} P_{e}\left(\gamma_{\mathrm{sr}}\right) \\
& +\left.P_{e}(\gamma)\right|_{\tilde{P}_{r}=P_{r}}\left(1-P_{e}\left(\gamma_{\mathrm{sr}}\right)\right),
\end{aligned}
$$

where $\gamma_{\mathrm{sr}}=P_{s} l_{\mathrm{sr}}^{-\alpha} \xi_{\mathrm{sr}}\left|h_{\mathrm{sr}}\right|^{2} / N_{0}$ is the SNR at the relay node. Substituting (4) and (5) into (6), the SER conditioned on the channel coefficients can be obtained. Since the channel involves not only small-scale fading but also shadowing, we should average the conditional SER over the small-scale fading and shadowing.

First, we average the conditional SER over the i.i.d. small-scale fading and obtain the SER conditioned on the shadowing as $[15,16]$

$$
\begin{aligned}
P_{e}\left(\xi_{\mathrm{sr}}, \xi_{\mathrm{sd}}, \xi_{\mathrm{rd}}\right)= & \Phi\left(1+\frac{\bar{\gamma}_{\mathrm{sd}} \sin ^{2}(\pi / M)}{\sin ^{2} \theta}\right) \times \Phi\left(1+\frac{\bar{\gamma}_{\mathrm{sr}} \sin ^{2}(\pi / M)}{\sin ^{2} \theta}\right) \\
& +\Phi\left[\left(1+\frac{\bar{\gamma}_{\mathrm{sd}} \sin ^{2}(\pi / M)}{\sin ^{2} \theta}\right)\left(1+\frac{\bar{\gamma}_{\mathrm{rd}} \sin ^{2}(\pi / M)}{\sin ^{2} \theta}\right)\right] \\
& \times\left[1-\Phi\left(1+\frac{\bar{\gamma}_{\mathrm{sr}} \sin ^{2}(\pi / M)}{\sin ^{2} \theta}\right)\right],
\end{aligned}
$$

where $\bar{\gamma}_{\mathrm{sd}}=P_{s} l_{\mathrm{sd}}^{-\alpha} \xi_{\mathrm{sd}} / N_{0}, \bar{\gamma}_{\mathrm{sr}}=P_{s} l_{\mathrm{sr}}^{-\alpha} \xi_{\mathrm{sr}} / N_{0}, \bar{\gamma}_{\mathrm{rd}}=$ $P_{r} l_{\mathrm{rd}}^{-\alpha} \xi_{\text {rd }} / N_{0}$, and

$$
\Phi(x(\theta))=\frac{1}{\pi} \int_{0}^{(M-1) \pi / M} \frac{1}{x(\theta)} d \theta .
$$

Next, we can average (7) over the correlated shadowing and obtain the SER performance. However, there is no closed-form expression for the SER, so we present an SER upper bound to provide some insights into the impacts of the correlated shadowing. In the SER expression (7), by removing the negative term and ignoring all 1 in the denominator, we have

$$
\begin{aligned}
P_{e}\left(\xi_{\mathrm{sr}}, \xi_{\mathrm{sd}}, \xi_{\mathrm{rd}}\right) \leq & \frac{A^{2}}{\bar{\gamma}_{\mathrm{sd}} \bar{\gamma}_{\mathrm{sr}} \sin ^{4}(\pi / M)} \\
& +\frac{B N_{0}^{2}}{\bar{\gamma}_{\mathrm{sd}} \bar{\gamma}_{\mathrm{rd}} \sin ^{4}(\pi / M)},
\end{aligned}
$$

where

$$
\begin{aligned}
A & =\frac{1}{\pi} \int_{0}^{(M-1) \pi / M} \sin ^{2} \theta d \theta=\frac{M-1}{2 M}+\frac{\sin (2 \pi / M)}{4 \pi}, \\
B & =\frac{1}{\pi} \int_{0}^{(M-1) \pi / M} \sin ^{4} \theta d \theta \\
& =\frac{3(M-1)}{8 M}+\frac{\sin (2 \pi / M)}{4 \pi}-\frac{\sin (4 \pi / M)}{32 \pi} .
\end{aligned}
$$

Thus, the SER upper bound can be obtained by averaging (9) over the correlated shadowing, i.e.,

$$
\begin{aligned}
P_{e} \leq & E_{\xi_{\mathrm{sr}}, \xi_{\mathrm{sd}}, \xi_{\mathrm{rd}}}\left(\frac{A^{2} N_{0}^{2}}{\bar{\gamma}_{\mathrm{sd}} \bar{\gamma}_{\mathrm{sr}} \sin ^{4}(\pi / M)}+\frac{B N_{0}^{2}}{\bar{\gamma}_{\mathrm{sd}} \bar{\gamma}_{\mathrm{rd}} \sin ^{4}(\pi / M)}\right) \\
= & \frac{A^{2} N_{0}^{2}}{P_{s}^{2} l_{\mathrm{sd}}^{-\alpha} l_{\mathrm{sr}}^{-\alpha} \sin ^{4}(\pi / M)} E_{\xi_{\mathrm{ss}}, \xi_{\mathrm{sd}}}\left(\frac{1}{\xi_{\mathrm{sd}} \xi_{\mathrm{sr}}}\right) \\
& +\frac{B N_{0}^{2}}{P_{s} l_{\mathrm{sd}}^{-\alpha} P_{r} l_{\mathrm{rd}}^{-\alpha} \sin ^{4}(\pi / M)} E_{\xi_{\mathrm{sd}}, \xi_{\mathrm{rd}}}\left(\frac{1}{\xi_{\mathrm{sd}} \xi_{\mathrm{rd}}}\right),
\end{aligned}
$$

where $E_{\xi_{\mathrm{sr}}, \xi_{\text {sd }}, \xi_{\text {rd }}}$ denotes the expectation with respect to $\xi_{\text {sr }}, \xi_{\text {sd }}$, and $\xi_{\text {rd }}$. With the assumption that $\xi_{\text {sr }}, \xi_{\text {sd }}$, and $\xi_{\text {rd }}$ are lognormal-distributed, $\frac{1}{\xi_{\mathrm{sd}} \xi_{\mathrm{sr}}}$ and $\frac{1}{\xi_{\mathrm{sd}} \xi_{\mathrm{rd}}}$ are also lognormal random variables, with the distribution

$$
\begin{aligned}
& 10 \log _{10}\left(\frac{1}{\xi_{\mathrm{sd}} \xi_{\mathrm{sr}}}\right) \sim N\left(0, \sigma_{\mathrm{sd}}^{2}+\sigma_{\mathrm{sr}}^{2}+2 \rho_{1} \sigma_{\mathrm{sd}} \sigma_{\mathrm{sr}}\right), \\
& 10 \log _{10}\left(\frac{1}{\xi_{\mathrm{sd}} \xi_{\mathrm{rd}}}\right) \sim N\left(0, \sigma_{\mathrm{sd}}^{2}+\sigma_{\mathrm{rd}}^{2}+2 \rho_{2} \sigma_{\mathrm{sd}} \sigma_{\mathrm{rd}}\right),
\end{aligned}
$$

whose mean values are given as

$$
\begin{aligned}
& E_{\xi_{\mathrm{sd}}, \xi_{\mathrm{sr}}}\left(\frac{1}{\xi_{\mathrm{sd}} \xi_{\mathrm{sr}}}\right)=\exp \left(\frac{\sigma_{\mathrm{sd}}^{2}+\sigma_{\mathrm{sr}}^{2}+2 \rho_{1} \sigma_{\mathrm{sd}} \sigma_{\mathrm{sr}}}{2 \eta^{2}}\right) \\
& E_{\xi_{\mathrm{sd}}, \xi_{\mathrm{rd}}}\left(\frac{1}{\xi_{\mathrm{sd}} \xi_{\mathrm{rd}}}\right)=\exp \left(\frac{\sigma_{\mathrm{sd}}^{2}+\sigma_{\mathrm{rd}}^{2}+2 \rho_{2} \sigma_{\mathrm{sd}} \sigma_{\mathrm{rd}}}{2 \eta^{2}}\right)
\end{aligned}
$$

with $\eta=10 / \ln 10$. By substituting (13) into (11), the SER upper bound considering the effects of path loss, correlated shadowing and flat Rayleigh fading, is given as

$$
\begin{aligned}
P_{e, \text { upper }}= & \frac{N_{0}^{2}}{\sin ^{4}(\pi / M)} \exp \left(\frac{\sigma_{\mathrm{sd}}^{2}}{2 \eta^{2}}\right) \\
& \times\left[\frac{A^{2} l_{\mathrm{sd}}^{\alpha} \mathrm{sr}_{\mathrm{sr}}^{\alpha}}{P_{s}^{2}} \exp \left(\frac{\sigma_{s r}^{2}+2 \rho_{1} \sigma_{s d} \sigma_{s r}}{2 \eta^{2}}\right)\right. \\
& \left.+\frac{B l_{\mathrm{sd}}^{\alpha} l_{\mathrm{rd}}^{\alpha}}{P_{s} P_{r}} \exp \left(\frac{\sigma_{\mathrm{rd}}^{2}+2 \rho_{2} \sigma_{\mathrm{sd}} \sigma_{\mathrm{rd}}}{2 \eta^{2}}\right)\right] .
\end{aligned}
$$

From (14), we can see that the SER upper bound is determined by not only the distances between these nodes but also the correlation coefficients and standard deviations of shadowing. 


\section{Optimization of power allocation and relay placement}

In this section, we optimize the power allocation and the relay placement to minimize the obtained SER upper bound. To provide some insights into the impacts of the correlated shadowing, we make the following three assumptions: (1) the locations of the source and the destination are fixed; (2) the source, relay, and destination are located on a straight line, i.e., $l_{\mathrm{sr}}+l_{\mathrm{rd}}=l_{\mathrm{sd}}$; (3) the total transmitted power of the source and the relay is $P_{t}$, i.e., $P_{s}+P_{r}=P_{t}$. Then, by substituting $l_{\mathrm{rd}}=l_{\mathrm{sd}}-l_{\mathrm{sr}}$ and $P_{r}=P_{t}-P_{s}$ into (14), the SER upper bound can be written as

$$
\begin{aligned}
P_{e, \text { upper }}= & G\left[\frac{A^{2} z^{\alpha}}{\beta^{2}} \exp \left(\frac{\sigma_{\mathrm{sr}}^{2}+2 \rho_{1} \sigma_{\mathrm{sd}} \sigma_{\mathrm{sr}}}{2 \eta^{2}}\right)\right. \\
& \left.+\frac{B(1-z)^{\alpha}}{\beta(1-\beta)} \exp \left(\frac{\sigma_{\mathrm{rd}}^{2}+2 \rho_{2} \sigma_{\mathrm{sd}} \sigma_{\mathrm{rd}}}{2 \eta^{2}}\right)\right],
\end{aligned}
$$

where $\beta=P_{s} / P_{t}, z=l_{\mathrm{sr}} / l_{\mathrm{sd}}$, and

$$
G=\left(\frac{N_{0}}{P_{t} l_{\mathrm{sd}}^{-\alpha} \sin ^{2}(\pi / M)}\right)^{2} \exp \left(\frac{\sigma_{\mathrm{sd}}^{2}}{2 \eta^{2}}\right) .
$$

The optimization problem can be formulated for the following three cases.

\subsection{Relay placement under fixed power allocation}

In this case, we derive the optimal relay placement under a predetermined power allocation, i.e., $\beta$ is fixed. The optimization problem can be formulated as

$$
\left\{\begin{array}{l}
z^{*}=\arg \min _{z} P_{e, \text { upper }} \\
\text { subject to } 0<z<1 .
\end{array}\right.
$$

By letting the derivative of $P_{e \text {,upper }}$ with respect to $z$ be equal to zero, we obtain the unique solution as

$$
z^{*}=\frac{1}{1+\left(\frac{W A^{2}(1-\beta)}{B \beta}\right)^{\frac{1}{\alpha-1}}},
$$

where

$$
W=\exp \left(\frac{\sigma_{\mathrm{sr}}^{2}+2 \rho_{1} \sigma_{\mathrm{sd}} \sigma_{\mathrm{sr}}-\sigma_{\mathrm{rd}}^{2}-2 \rho_{2} \sigma_{\mathrm{sd}} \sigma_{\mathrm{rd}}}{2 \eta^{2}}\right) .
$$

Taking the second derivative of $P_{e, \text { upper }}$ with respect to $z$, we have

$$
\begin{aligned}
\frac{\partial^{2} P_{e, \text { upper }}}{\partial z^{2}}= & G\left[\frac{\alpha(\alpha-1) A^{2} z^{\alpha-2}}{\beta^{2}} \exp \left(\frac{\sigma_{\mathrm{sr}}^{2}+2 \rho_{1} \sigma_{\mathrm{sd}} \sigma_{\mathrm{sr}}}{2 \eta^{2}}\right)\right. \\
& \left.+\frac{\alpha(\alpha-1) B(1-z)^{\alpha-2}}{\beta(1-\beta)} \exp \left(\frac{\sigma_{\mathrm{rd}}^{2}+2 \rho_{2} \sigma_{\mathrm{sd}} \sigma_{\mathrm{rd}}}{2 \eta^{2}}\right)\right] .
\end{aligned}
$$

It is easy to see that $\frac{\partial^{2} P_{e, \text { upper }}}{\partial z^{2}}>0$ for $\alpha>1$ and $0<z<1$, which means the objective function is convex [17]. Therefore, we conclude that the solution given in (18) is optimal to problem (17). From (18) and (19), it is observed that the optimal relay placement depends on the correlation coefficients and standard deviations of shadowing, the power allocation strategy, the path loss exponent, and the modulation schemes. When the auto-correlation coefficient or the standard deviation over source-to-relay increases, the relay should be placed more closely to the source. On the other hand, when the crosscorrelation coefficient or the standard deviation over relay-to-destination increases, the relay should be placed more closely to the destination. Since a larger correlation coefficient or standard deviation of shadowing means worse link quality, we conclude that the relay should be placed more closely to the node with a larger correlation coefficient and standard deviation of shadowing so as to compensate for the loss of the link quality.

\subsection{Power allocation under fixed relay placement}

In this case, we derive the optimal power allocation by assuming fixed relay placement, i.e., $z$ is fixed. The optimization problem can be formulated as

$$
\left\{\begin{array}{l}
\beta^{*}=\arg \min _{\beta} P_{e, \text { upper }} \\
\text { subject to } 0<\beta<1 .
\end{array}\right.
$$

Similarly, taking the first derivative of $P_{e \text {,upper }}$ with respect to $\beta$ and setting it to zero, we obtain the unique solution as

$$
\beta^{*}=\frac{1+\sqrt{1+\frac{8 A^{2} z^{\alpha} W}{B(1-z)^{\alpha}}}}{3+\sqrt{1+\frac{8 A^{2} z^{\alpha} W}{B(1-z)^{\alpha}}}} .
$$

Moreover, the second derivative of $P_{e \text {,upper with respect }}$ to $\beta$ is

$$
\begin{aligned}
& \frac{\partial^{2} P_{e, \text { upper }}}{\partial \beta^{2}}=G\left[\frac{6 A^{2} z^{\alpha}}{\beta^{4}} \exp \left(\frac{\sigma_{\mathrm{sr}}^{2}+2 \rho_{1} \sigma_{\mathrm{sd}} \sigma_{\mathrm{sr}}}{2 \eta^{2}}\right)\right. \\
& \left.+\frac{2 B\left(3 \beta^{2}-3 \beta+1\right)}{\beta^{3}(1-\beta)^{3}(1-z)^{-\alpha}} \exp \left(\frac{\sigma_{\mathrm{rd}}^{2}+2 \rho_{2} \sigma_{\mathrm{sd}} \sigma_{\mathrm{rd}}}{2 \eta^{2}}\right)\right] .
\end{aligned}
$$

It can be seen that $\frac{\partial^{2} P_{e, \text { upper }}}{\partial \beta^{2}}>0$ for $0<\beta<1$. This implies that the objective function is convex and the solution given in (22) is optimal to problem (21). From (22), we observe that the correlation coefficients and standard deviations of shadowing and the relay placement have significant impacts on the optimal power allocation. As the auto-correlation coefficient or the standard deviation over the source-relay link increases, the power at the source should increase and the power at the relay should decrease. If the shadowing between source to relay is much more severe than that between relay to destination, i.e., $\sigma_{\mathrm{sr}} \gg \sigma_{\text {rd }}$, then $P_{s}$ goes to $P_{t}$ and $P_{r}$ goes to 0 , which 
means that we should put the total power at the source and do not need to use the relay. As the standard deviation over the relay-destination link increases, the power at the source should decrease and the power at the relay should increase. If the shadowing between relay to destination is much more severe than that between source to relay, i.e., $\sigma_{\text {sr }} \ll \sigma_{\text {rd }}$, then $P_{s}$ and $P_{r}$ both go to $P_{t} / 2$, which means that equal power allocation at the source and the relay should be adopted.

\subsection{Joint optimization of power allocation and relay placement}

In this subsection, we jointly optimize the power allocation and relay placement to further improve the SER performance. The optimization problem can be formulated as

$$
\left\{\begin{array}{l}
\left(\beta^{*}, z^{*}\right)=\arg \min _{\beta, z} P_{e, \text { upper }} \\
\text { subject to } 0<\beta<1 \text { and } 0<z<1 .
\end{array}\right.
$$

Since the matrix of the second partial derivative (Hessian matrix) [17] of the objective function stated in (15) is complex, it is difficult to obtain the necessary and sufficient conditions for the Hessian matrix to be positive definite. In the Appendix, we prove that the Hessian matrix is positive definite for $\alpha>3$, which is assumed in this paper for analytical tractability. Then, the optimal power allocation and relay placement can be obtained by setting the first derivatives of the objective function with respect to $\beta$ and $z$ to zero, which are given as

$$
\begin{aligned}
\frac{\partial P_{e, \text { upper }}}{\partial \beta}= & G\left[-\frac{2 A^{2} z^{\alpha}}{\beta^{3}} \exp \left(\frac{\sigma_{\mathrm{sr}}^{2}+2 \rho_{1} \sigma_{\mathrm{sd}} \sigma_{\mathrm{sr}}}{2 \eta^{2}}\right)\right. \\
& \left.-\frac{(1-2 \beta) B(1-z)^{\alpha}}{\beta^{2}(1-\beta)^{2}} \exp \left(\frac{\sigma_{\mathrm{rd}}^{2}+2 \rho_{2} \sigma_{\mathrm{sd}} \sigma_{\mathrm{rd}}}{2 \eta^{2}}\right)\right]=0,
\end{aligned}
$$

$$
\begin{aligned}
\frac{\partial P_{e, \text { upper }}}{\partial z}= & G\left[\frac{\alpha A^{2} z^{\alpha-1}}{\beta^{2}} \exp \left(\frac{\sigma_{\mathrm{sr}}^{2}+2 \rho_{1} \sigma_{\mathrm{sd}} \sigma_{\mathrm{sr}}}{2 \eta^{2}}\right)\right. \\
& \left.-\frac{\alpha B(1-z)^{\alpha-1}}{\beta(1-\beta)} \exp \left(\frac{\sigma_{\mathrm{rd}}^{2}+2 \rho_{2} \sigma_{\mathrm{sd}} \sigma_{\mathrm{rd}}}{2 \eta^{2}}\right)\right]=0 .
\end{aligned}
$$

After some mathematical manipulations of (25) and (26), we obtain the equivalent equations as

$$
\frac{(1-z)^{\alpha-2}(z+1)}{z^{\alpha-1}}=\frac{A^{2} W}{B} .
$$

$$
2 \beta-1=z .
$$

It is difficult to obtain the closed-form expression for the above equations. However, we note that (27) is an equation with only one variable, and thus, the numerical solution for $z$ can be obtained by using the root-finding algorithms such as the bisection method and Newton's method [17]. Thereafter, by substituting the obtained $z^{*}$ into (28), we obtain the optimal solution for $\beta$. From (28), it is observed that $0.5<\beta^{*}<1$ since $0<z<1$, which means that the equal power strategy is not optimal and more power should be allocated to the source node.

\section{Numerical and simulation results}

In this section, we present some numerical and simulation results to validate our analysis. For simplicity, we normalize the distance between the source and the destination to unity, i.e., $l_{\mathrm{sd}}=1$. The source and the relay use QPSK modulation for data transmission, and the path loss exponent $\alpha$ is set to 4 .

In Figure 2, we show the relationship between the relay placement and different standard deviations of shadowing, where the transmit power of the source and that of the relay are the same, i.e., $\beta=0.5$. Moreover, the correlation coefficients $\rho_{1}$ and $\rho_{2}$ are set to 0.5 , and $P_{t} / N_{0}$ is set to $25 \mathrm{~dB}$. We investigate three cases. It is assumed that $\sigma_{\mathrm{sd}}=\sigma_{\mathrm{sr}}=\sigma_{\mathrm{rd}}=6 \mathrm{~dB}$ in case I, $\sigma_{\mathrm{sd}}=\sigma_{\mathrm{rd}}=6$ $\mathrm{dB}$ and $\sigma_{\mathrm{sr}}=3 \mathrm{~dB}$ in case II, and $\sigma_{\mathrm{sd}}=\sigma_{\mathrm{sr}}=6 \mathrm{~dB}$ and $\sigma_{\mathrm{rd}}=3 \mathrm{~dB}$ in case III. The optimal values of $z^{*}$ are given in Table 1. It is observed from Figure 2 that when the standard deviations over source-relay and relaydestination links are equal, the optimal relay placement is $z^{*} \approx 0.55$. As the standard deviation of shadowing over the source-relay link decreases, the optimal relay location should be closer to the destination node. On the other hand, as the standard deviation of shadowing over the relay-destination link decreases, the optimal relay location should be closer to the source node.

Figure 3 shows the relationship between the relay power allocation and different standard deviations of shadowing, where the relay is located in the middle of the source and the destination, and other simulation parameters are the same as in Figure 2. The optimal values of $\beta^{*}$ are also given

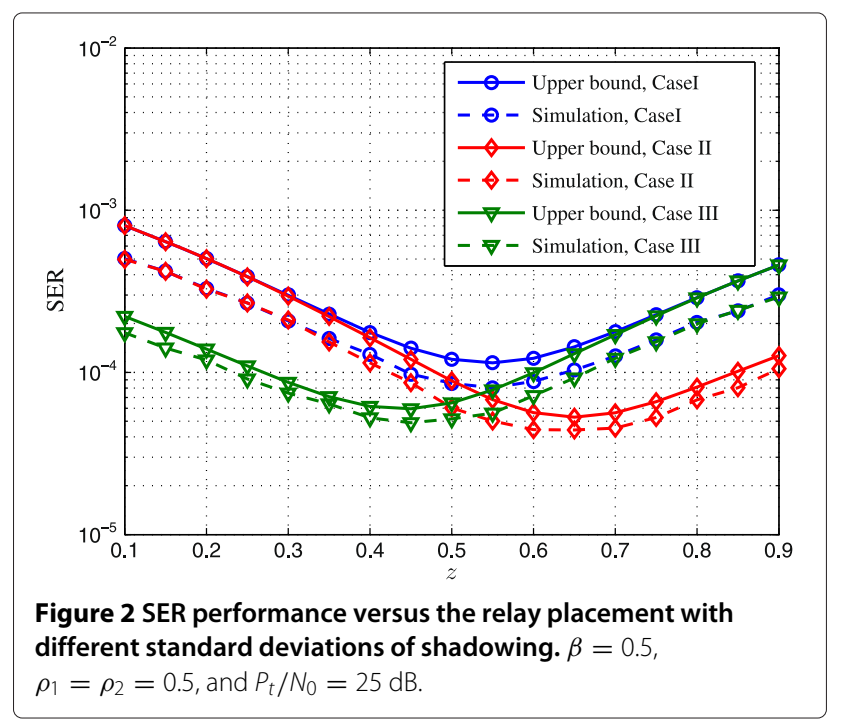


Table 1 The optimal values of $z^{*}$ and $\beta^{*}$ for different cases

\begin{tabular}{lccc}
\hline & Case I & Case II & Case III \\
\hline $\begin{array}{l}\text { Relay placement under } \\
\text { equal power allocation }\end{array}$ & $z^{*} \approx 0.55$ & $z^{*} \approx 0.65$ & $z^{*} \approx 0.45$ \\
$\begin{array}{l}\text { Power allocation under } \\
\text { midpoint relay placement }\end{array}$ & $\beta^{*} \approx 0.6$ & $\beta^{*} \approx 0.55$ & $\beta^{*} \approx 0.7$ \\
\hline
\end{tabular}

in Table 1. From Figure 3, we can observe that if the standard deviations over source-relay and relay-destination links are equal, the optimal power allocation factor is $\beta^{*} \approx$ 0.6 . As the standard deviation of shadowing over the relaydestination link decreases, we should put more power at the source. On the other hand, as the standard deviation of shadowing over the source-relay link decreases, we should put more power at the relay.

Then, we consider a special case with $\sigma_{\mathrm{sd}}=7 \mathrm{~dB}, \sigma_{\mathrm{sr}}=$ $3 \mathrm{~dB}, \sigma_{\mathrm{rd}}=5 \mathrm{~dB}, \rho_{1}=0.2$, and $\rho_{2}=0.6$. For this case, we can obtain the solution of (17) as $z^{*}=0.65$ for equal power allocation, the solution of (21) as $\beta^{*}=0.55$ for the relay locating in the middle of the source and the destination, and the solution of $(24)$ as $\left(\beta^{*}, z^{*}\right)=(0.895,0.79)$ for the joint optimization. In Figure 4, we compare the SER performance for different optimization schemes. It is observed from Figure 4 that joint optimization outperforms the other two optimization schemes and achieves the minimum SER. Moreover, the gap between the simulation results and the SER upper bound gets smaller with the increase of SNR, which means that the SER upper bound is asymptotically tight in the high SNR regime.

\section{Conclusion}

In this paper, we investigated the optimization of relay placement and power allocation by minimizing the SER upper bound for DF cooperative diversity over correlated

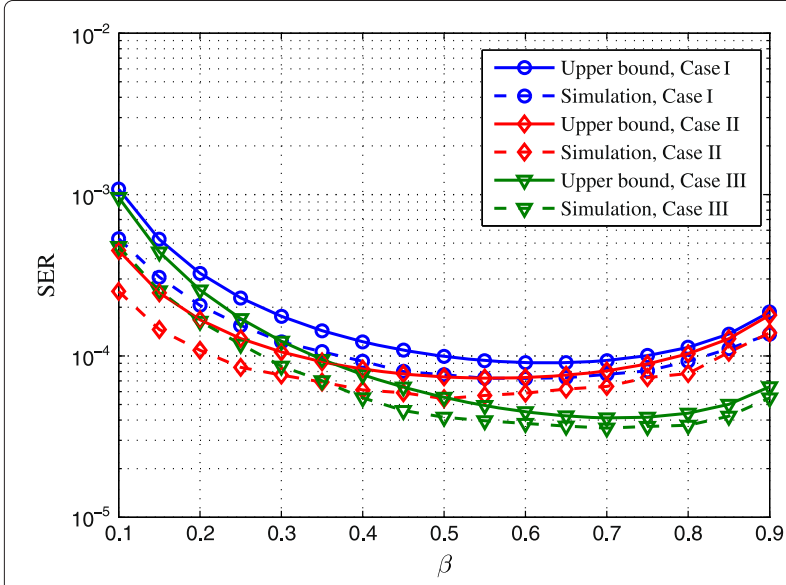

Figure 3 SER performance versus the power allocation factor with different standard deviations of shadowing. $z=0.5$, $\rho_{1}=\rho_{2}=0.5$, and $P_{t} / N_{0}=25 \mathrm{~dB}$.

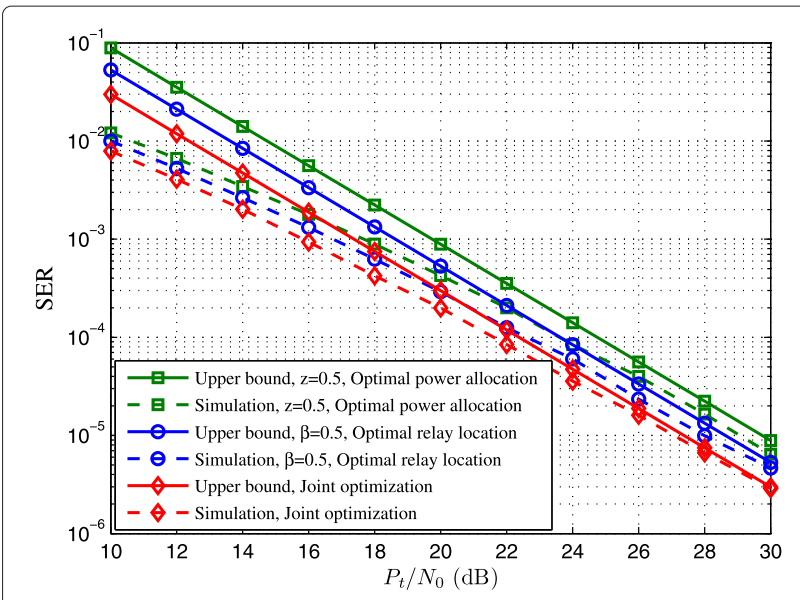

Figure 4 SER performance under different optimization

schemes. $\sigma_{\mathrm{sd}}=7 \mathrm{~dB}, \sigma_{\mathrm{sr}}=3 \mathrm{~dB}, \sigma_{\mathrm{rd}}=5 \mathrm{~dB}, \rho_{1}=0.2$, and $\rho_{2}=0.6$.

lognormal shadowed Rayleigh fading channels. It was shown that the correlation coefficients and standard deviations of shadowing have significant impacts on the SER performance and hence on the optimal relay placement and power allocation. We compared three optimization schemes and showed that joint optimization obtains the best SER performance. Our method provided a scheme to determine the optimal relay placement and power allocation for next-generation wireless communication networks.

\section{Appendix}

The SER upper bound in (15) can be written as

$$
\begin{aligned}
P_{e, \text { upper }}= & G\left[A^{2} \exp \left(\frac{\sigma_{\mathrm{sr}}^{2}+2 \rho_{1} \sigma_{\mathrm{sd}} \sigma_{\mathrm{sr}}}{2 \eta^{2}}\right) \cdot f(\beta, z)\right. \\
& \left.+B \exp \left(\frac{\sigma_{\mathrm{rd}}^{2}+2 \rho_{2} \sigma_{\mathrm{sd}} \sigma_{\mathrm{rd}}}{2 \eta^{2}}\right) \cdot g(\beta, z)\right],
\end{aligned}
$$

where

$$
\begin{aligned}
& f(\beta, z)=\frac{z^{\alpha}}{\beta^{2}}, \\
& g(\beta, z)=\frac{(1-z)^{\alpha}}{\beta(1-\beta)} .
\end{aligned}
$$

The Hessian matrix of $f(\beta, z)$ and $g(\beta, z)$ are given as

$$
\begin{aligned}
& \nabla^{2} f(\beta, z)=\left[\begin{array}{cc}
\frac{6 z^{\alpha}}{\beta^{4}} & -\frac{2 \alpha z^{\alpha-1}}{\beta^{3}} \\
-\frac{2 \alpha z^{\alpha-1}}{\beta^{3}} & \frac{\alpha(\alpha-1) z^{\alpha-2}}{\beta^{2}}
\end{array}\right], \\
& \nabla^{2} g(\beta, z)=\left[\begin{array}{cc}
\frac{2(1-z)^{\alpha}\left(3 \beta^{2}-3 \beta+1\right)}{\beta^{3}(1-\beta)^{3}} & \frac{\alpha(1-z)^{\alpha-1}(1-2 \beta)}{\beta^{2}(1-\beta)^{2}} \\
\frac{\alpha(1-z)^{\alpha-1}(1-2 \beta)}{\beta^{2}(1-\beta)^{2}} & \frac{\alpha(\alpha-1)(1-z)^{\alpha-2}}{\beta(1-\beta)}
\end{array}\right] .
\end{aligned}
$$


Then, the determinant of the above Hessian matrices are given as

$$
\begin{aligned}
& \operatorname{det}\left[\nabla^{2} f(\beta, z)\right]=\frac{2 \alpha(\alpha-3) z^{2 \alpha-2}}{\beta^{6}}, \\
& \operatorname{det}\left[\nabla^{2} g(\beta, z)\right]=\frac{\alpha\left[(2 \alpha-6) \beta^{2}+(6-2 \alpha) \beta+\alpha-2\right]}{(1-z)^{2-2 \alpha} \beta^{4}(1-\beta)^{4}} .
\end{aligned}
$$

It is easy to check that the above determinants are positive for $\alpha>3$, which means that the Hessian matrix of $f(\beta, z)$ and $g(\beta, z)$ are positive definite for $\alpha>3$. Therefore, the Hessian matrix of the objective function stated in (15) is positive definite for $\alpha>3$.

\section{Competing interests}

The authors declare that they have no competing interests.

\section{Acknowledgements}

This work was supported by the National Natural Science Foundation of China (61372097, 61271411).

Received: 27 February 2014 Accepted: 3 March 2014

Published: 18 March 2014

\section{References}

1. Y Yang, H Hu, J Xu, G Mao, Relay technologies for WiMAX and LTE-Advanced mobile systems. IEEE Commun. Mag. 47(10), 100-105 (2009)

2. C Hoymann, W Chen, J Montojo, A Golitschek, C Koutsimanis, X Shen, Relaying operation in 3GPP LTE: challenges and solutions. IEEE Commun. Mag. 50(2), 156-162 (2012)

3. J Laneman, D Tse, G Wornell, Cooperative diversity in wireless networks: efficient protocols and outage behavior. IEEE Trans. Inf. Theory. 50(12) 3062-3080 (2004)

4. G Kramer, M Gastpar, P Gupta, Cooperative strategies and capacity theorems for relay networks. IEEE Trans. Inf. Theory 51(9), 3037-3063 (2005)

5. R Nabar, H Bölcskei, F Kneubühler, Fading relay channels: performance limits and space-time signal design. IEEE J. Select. Areas Commun. 22(6), 1099-1109 (2004)

6. A Nasri, R Schober, I Blake, Performance and optimization of amplify-and-forward cooperative diversity systems in generic noise and interference. IEEE Trans. Wireless Commun. 10(4), 1132-1143 (2011)

7. H Yu, I Lee, G Stüber, Outage probability of decode-and-forward cooperative relaying systems with co-channel interference. IEEE Trans. Wireless Commun. 11(1), 266-274 (2012)

8. A Sadek, Z Han, K Ray Liu, Distributed relay-assignment protocols for coverage expansion in cooperative wireless networks. IEEE Trans. Mobile Comput. 9(4), 505-515 (2011)

9. P Agrawal, N Patwari, Correlated link shadow fading in multi-hop wireless networks. IEEE Trans. Wireless Commun. 8(8), 4024-4036 (2009)

10. S Szyszkowicz, H Yanikomeroglu, J Thompson, On the feasibility of wireless shadowing correlation models. IEEE Trans. Veh. Technol. 59(9), 4222-4236 (2010)

11. M Safari, M Uysal, Cooperative diversity over log-normal fading channels: performance analysis and optimization. IEEE Trans. Wireless Commun. 7(5), 1963-1372 (2008)

12. M Renzo, F Graziosi, F Santucci, A comprehensive framework for performance analysis of cooperative multi-hop wireless systems over log-normal fading channels. IEEE Trans. Commun. 58(2), 531-544 (2010)

13. V Sakarellos, D Skraparlis, A Panagopoulos, Cooperative radio communications over correlated shadowing: outage analysis. Paper presented at future network \& mobile summit (FutureNetw) (Berlin, Germany, 4-6 July 2012), pp. 1-8
14. D Tse, P Viswanath, Fundamentals of Wireless Communication (Cambridge University Press, Cambridge, 2005)

15. M Simon, M Alouini, Digital Communication over Fading Channels: A Unified Approach to Performance Analysis (Wiley, New York, 2000)

16. KRay Liu, A Sadek, W Su, A Kwasinski, Cooperative Communications Networking (Cambridge University Press, Cambridge, 2009)

17. S Boyd, L Venberghe, Convex Optimization (Cambridge University Press, Cambridge, 2004)

doi:10.1186/1687-1499-2014-41

Cite this article as: Han et al:: Optimization of relay placement and power allocation for decode-and-forward cooperative relaying over correlated shadowed fading channels. EURASIP Journal on Wireless Communications and Networking 2014 2014:41.

\section{Submit your manuscript to a SpringerOpen ${ }^{\circ}$ journal and benefit from:}

- Convenient online submission

- Rigorous peer review

- Immediate publication on acceptance

- Open access: articles freely available online

- High visibility within the field

- Retaining the copyright to your article

Submit your next manuscript at $>$ springeropen.com 